\title{
New bounds for equiangular lines
}

\author{
Alexander Barg and Wei-Hsuan Yu \\ To Ilya Dumer, on the occasion of his 60th birthday
}

\begin{abstract}
A set of lines in $\mathbb{R}^{n}$ is called equiangular if the angle between each pair of lines is the same. We address the question of determining the maximum size of equiangular line sets in $\mathbb{R}^{n}$, using semidefinite programming to improve the upper bounds on this quantity. Improvements are obtained in dimensions $24 \leq n \leq 136$. In particular, we show that the maximum number of equiangular lines in $\mathbb{R}^{n}$ is 276 for all $24 \leq n \leq 41$ and is 344 for $n=43$. This provides a partial resolution of the conjecture set forth by Lemmens and Seidel (1973).
\end{abstract}

\section{Introduction}

A set of lines in a metric space is called equiangular if the angle between each pair of lines is the same. We are interested in upper bounds on the number of equiangular lines in $\mathbb{R}^{n}$. In other words, if we have a set of unit vectors $S=\left\{x_{i}\right\}_{i=1}^{M}$ and there is a constant $c>0$ such that $\left|\left\langle x_{i}, x_{j}\right\rangle\right|=c$ for all $1 \leq i \neq j \leq M$, what is the maximum cardinality of $S$ ? Denote this quantity by $M(n)$. The problem of determining $M(n)$ looks elementary but a general answer has so far proved elusive: Until recently the maximum number of equiangular lines in $\mathbb{R}^{n}$ was known only for 16 values of the dimension $n$. The history of this problem started with Hanntjes 8 who found $M(n)$ for $n=2$ and 3 in 1948. Van Lint and Seidel 11 found the largest number of equiangular lines for $4 \leq n \leq 7$. In 1973, Lemmens and Seidel 10] used linear-algebraic methods to determine $M(n)$ for most values of $n$ in the region $8 \leq n \leq 23$. Gerzon (see [10]) gave the following upper on $M(n)$.

Theorem 1.1 (Gerzon). If there are $M$ equiangular lines in $\mathbb{R}^{n}$, then

$$
M \leq \frac{n(n+1)}{2}
$$

Gerzon's upper bound can be attained only for a very small number of values of $n$. Currently, such constructions are known only for $n=2,3,7$, and 23. Neumann (see [10, Theorem 3.2) proved a fundamental result in this area:

2000 Mathematics Subject Classification. Primary 52C35; Secondary $94 \mathrm{~B} 75$.

Key words and phrases. Semidefinite Programming, Two-Distance Sets, Tight Designs.

(C) 0000 (copyright holder) 


\begin{tabular}{|c|c|c|c|c|c|}
\hline$n$ & $M(n)$ & $1 / \alpha$ & $n$ & $M(n)$ & $1 / \alpha$ \\
\hline 2 & 3 & 2 & 17 & $48-50$ & 5 \\
3 & 6 & $\sqrt{5}$ & 18 & $48-61$ & 5 \\
4 & 6 & $3 ; \sqrt{5}$ & 19 & $72-76$ & 5 \\
5 & 10 & 3 & 20 & $90-96$ & 5 \\
6 & 16 & 3 & 21 & 126 & 5 \\
$7 \leq n \leq 13$ & 28 & 3 & 22 & 176 & 5 \\
14 & $28-30$ & $3 ; 5$ & 23 & 276 & 5 \\
15 & 36 & 5 & $24 \leq n \leq 42$ & $\geq 276$ & 5 \\
16 & $40-42$ & 5 & 43 & $\geq 344$ & 7 \\
\hline
\end{tabular}

TABLE 1. Known bounds on $M(n)$ in small dimensions

Theorem 1.2 (Neumann). If there are $M$ equiangular lines in $\mathbb{R}^{n}$ with angle $\arccos \alpha$ and $M>2 n$, then $1 / \alpha$ is an odd integer.

Note that if $M$ attains the Gerzon bound, then $(n+2) \alpha^{2}=1$ 10, Thm.3.5]. Therefore, if the cardinality of an equiangular line set attains the Gerzon bound, then $n$ has to be 2 or 3 or an odd square minus two and the angle between pairs of lines is $\arccos 1 /(\sqrt{n+2})$.

A set of unit vectors $S=\left\{x_{1}, x_{2}, \ldots\right\} \subset \mathbb{R}^{n}$ is called two-distance if $\left\langle x_{i}, x_{j}\right\rangle \in$ $\{a, b\}$ for some $a, b$ and all $i \neq j$.

Theorem 1.3 (Larman, Rogers, and Seidel $\mathbf{9}$ ). Let $S$ be a spherical twodistance set in $\mathbb{R}^{n}$. If $|S|>2 n+3$ and $a>b$, then $b=\frac{k a-1}{k-1}$ for some integer $k$ such that $2 \leq k \leq(1+\sqrt{2 n}) / 2$.

The condition $|S|>2 n+3$ was improved to $|S|>2 n+1$ by Neumaier $\mathbf{1 3}$. He also gave an example of a two-distance set with cardinality $2 n+1$ that violates the integeraity condition of $k$. This example is obtained from the spherical embedding of the conference graph.

If the spherical two-distance set gives rise to equiangular lines, then $a=-b$, so Theorem 1.3 implies that $a=1 /(2 k-1)$, which is the statement of the Neumann theorem. The assumption of Theorem 1.3 is more restrictive than of Theorem 1.2 , but in return we obtain an upper bound on $k$. For instance, if $n=40$, then $k$ can be only 2 or 3 , so the angle has to be $\arccos \alpha$, where $\alpha=1 / 3$ or $1 / 5$. The assumption of Theorem 1.3 is satisfied since there exist equiangular line sets with $M \geq 2 n+4$ for all $n \geq 15$.

The known bounds on $M(n)$ for small dimensions are summarized in Table 11 [10, 15. (the latter for the upper bound on $M(17)$ ); in particular, $M(n)$ was known exactly only if $2 \leq n \leq 13 ; n=15,21,22,23$. In the unsettled cases the best known upper bound on $M(n)$ is usually the Gerzon bound. Lemmens and Seidel [10, Thm. 4.5] further showed that

$$
M_{1 / 3}(n) \leq 2(n-1), \quad n \geq 16,
$$

where $M_{\alpha}(n)$ is the maximum size of an equiangular line set when the value of the angle is $\arccos \alpha$. They also conjectured that $M_{1 / 5}(n)=276$ for $23 \leq n \leq 185$, observing that if this conjecture is true, then $M(n)=276$ for $24 \leq n \leq 41$ and 


\begin{tabular}{|c|c|c|c|c|c|}
\hline$n$ & $M(n)$ & SDP bound & $n$ & $M(n)$ & SDP bound \\
\hline 3 & 6 & 6 & 18 & $48-61$ & 61 \\
\hline 4 & 6 & 6 & 19 & $72-76$ & 76 \\
\hline 5 & 10 & 10 & 20 & $90-96$ & 96 \\
\hline 6 & 16 & 16 & 21 & 126 & 126 \\
\hline $7 \leq n \leq 13$ & 28 & 28 & 22 & 176 & 176 \\
\hline 14 & $28-30$ & 30 & 23 & 276 & 276 \\
\hline 15 & 36 & 36 & $24 \leq n \leq 41$ & 276 & 276 \\
\hline 16 & $40-42$ & 42 & 42 & $\geq 276$ & 288 \\
\hline 17 & $48-50$ & 51 & 43 & 344 & 344 \\
\hline
\end{tabular}

TABLE 2. Bounds on $M(n)$ including new results

$M(43)=344$. Note that generally we have [10]:

$$
M_{\alpha}(n) \leq \frac{n\left(1-\alpha^{2}\right)}{1-n \alpha^{2}}
$$

valid for all $\alpha$ such that the denominator is positive. This inequality is sometimes called the relative bound as opposed to the "absolute bound" of (1.1).

In this paper we use the semidefinite programming (SDP) method to derive some new bounds on $M(n)$. Our main results are summarized in Table 2. In particular, exact values of $M(n)$ are obtained for $24 \leq n \leq 41$ and for $n=43$ where previous results gave divergent bounds: we show that $M(n)=276$ for $24 \leq n \leq 41$ and $M(43)=344$. These results are established by performing computations with SDP. We also show that $M_{1 / 5}(n)=276$ for $23 \leq n \leq 60$. These results resolve a part of the Lemmens-Seidel conjecture and enable us to obtain the results in Table 2. For $44 \leq n \leq 136$, we also obtain new upper bounds on $M(n)$, improving upon the Gerzon bound, although no new exact values are found in this range. Below in the paper we give a more complete table of the computation results.

An interesting question relates to the asymptotic behavior of $M(n)$ for $n \rightarrow \infty$. For a long time the best known constructions were able to attain the growth order of $M(n)=\Omega(n)$, until D. de Caen [6] constructed a family of $\frac{2}{9}(n+1)^{2}$ equiangular lines in $\mathbb{R}^{n}$ for $n=3 \cdot 2^{2 t-1}, t \in \mathbb{N}$. Thus, currently the best asymptotic results are summarized as follows:

$$
\frac{2}{9} \leq \limsup _{n \rightarrow \infty} \frac{M(n)}{n^{2}} \leq \frac{1}{2},
$$

where the upper bound is from (1.1). The question of the correct order of growth represents a difficult unresolved problem. Contributing to the study of the asymptotic bounds, we show that for $n=3(2 k-1)^{2}-4$ and $\alpha=\frac{1}{2 k-1}$, for all integer $k \geq 2$,

$$
M_{\alpha}(n) \leq \frac{(n+1)(n+2)}{6}
$$

(ADDED IN PROOF) After this paper was accepted, C. Greaves et al. posted a preprint [16 in which the upper bounds for $n=14,16$ were improved to $M(14) \leq 29$ and $M(16) \leq 41$, respectively. 


\section{SDP bounds for equiangular lines}

Many problems in operations research, combinatorial optimization, control theory, and discrete geometry can be modelled or approximated as semidefinite programming. SDP optimization problems are usually stated in the following form:

$$
\begin{gathered}
\min c^{T} x \\
\text { subject to } \quad F_{0}+\sum_{i=1}^{m} F_{i} x_{i} \succeq 0, \quad x \in \mathbb{R}^{m},
\end{gathered}
$$

where $c \in \mathbb{R}^{m}$ is a given vector of coefficients, $F_{i}, i=0,1, \ldots$ are $n \times n$ symmetric matrices, and " $\succeq$ " means that the matrix is positive semidefinite. SDP problems fall in the class of convex optimization problems since the domain of feasible solutions is a convex subset of $\mathbb{R}^{m}$. For the case of diagonal matrices $F_{i}$, SDP turns into a linear programming (LP) problem. Properties of SDP problems and algorithms for their solution are discussed, for instance, in [5. Most SDP solvers such as CSDP, Sedumi, SDPT3 use interior point methods originating with Karmarkar's celebrated algorithm (we used CVX toolbox in Matlab).

Let $\mathcal{C} \subset S^{n-1}$ be a set of unit vectors in $\mathbb{R}^{n}$ such that $\left\langle x, x^{\prime}\right\rangle \leq a$ for all $x, x^{\prime} \in \mathcal{C}, x \neq x^{\prime}$ (a spherical code). As shown by Bachoc and Vallentin [1, the problem of estimating the maximum size of $\mathcal{C}$ can be stated as an SDP problem. In particular, for $a=1 / 2$, this is the famous "kissing number problem", i.e., the question about the maximum number of nonoverlapping unit spheres that can touch a given unit sphere. A particular case of the main result in [1] was used in [4] to find new bounds on the maximum cardinality of spherical two-distance sets.

Let us introduce some notation. Let $G_{k}^{(n)}(t), k=0,1, \ldots$ denote the Gegenbauer polynomials of degree $k$, i.e., a family of polynomials defined recursively as follows: $G_{0}^{(n)} \equiv 1, G_{1}^{(n)}(t)=t$, and

$$
G_{k}^{(n)}(t)=\frac{(2 k+n-4) t G_{k-1}^{(n)}(t)-(k-1) G_{k-2}^{(n)}(t)}{k+n-3}, \quad k \geq 2 .
$$

Following [1], define a $(p-k+1) \times(p-k+1)$ matrix $Y_{k}^{n}(u, v, t), k \geq 0$,

$$
\left(Y_{k}^{n}(u, v, t)\right)_{i j}=u^{i} v^{j}\left(\left(1-u^{2}\right)\left(1-v^{2}\right)\right)^{k / 2} G_{k}^{(n-1)}\left(\frac{t-u v}{\sqrt{\left(1-u^{2}\right)\left(1-v^{2}\right)}}\right),
$$

where $p \in \mathbb{N}$, and a matrix $S_{k}^{n}(u, v, t)$ by setting

$$
S_{k}^{n}(u, v, t)=\frac{1}{6} \sum_{\sigma} Y_{k}^{n}(\sigma(u, v, t))
$$

where the sum is over all permutations of 3 elements. Note that $\left(S_{k}^{n}(1,1,1)\right)_{i j}=0$ for all $i, j$ and all $k \geq 1$. Let $\mathcal{C}$ be a spherical code. As shown in [7],

$$
\sum_{(x, y) \in \mathcal{C}^{2}} G_{k}^{(n)}(\langle x, y\rangle) \geq 0
$$

and as shown in [1,

$$
\sum_{(x, y, z) \in \mathcal{C}^{3}} S_{k}^{n}(\langle x, y\rangle,\langle x, z\rangle,\langle y, z\rangle) \succeq 0 .
$$

Inequalities (2.1) and (2.2) can be used to formulate a general SDP problem for upper bounds on the cardinality of spherical codes in $\mathbb{R}^{n}[\mathbf{1}$. 
Using the approach of $\underline{4}$, we obtain the following SDP bound on $M(n)$.

THEOREM 2.1. Let $\mathcal{C}$ be set of a equiangular lines with inner product values either $a$ or $-a$. Let $p$ be the positive integer. The cardinality $|\mathcal{C}|$ is bounded above by the solution of the following semi-definite programming problem:

$$
1+\frac{1}{3} \max \left(x_{1}+x_{2}\right)
$$

subject to

$$
\begin{array}{r}
\left(\begin{array}{ll}
1 & 0 \\
0 & 1
\end{array}\right)+\frac{1}{3}\left(\begin{array}{ll}
0 & 1 \\
1 & 1
\end{array}\right)\left(x_{1}+x_{2}\right)+\left(\begin{array}{ll}
0 & 0 \\
0 & 1
\end{array}\right)\left(x_{3}+x_{4}+x_{5}+x_{6}\right) \succeq 0 \\
S_{k}^{n}(1,1,1)+S_{k}^{n}(a, a, 1) x_{1}+S_{k}^{n}(-a,-a, 1) x_{2}+S_{k}^{n}(a, a, a) x_{3} \\
+S_{k}^{n}(a, a,-a) x_{4}+S_{k}^{n}(a,-a,-a) x_{5}+S_{k}^{n}(-a,-a,-a) x_{6} \succeq 0 \\
3+G_{k}^{(n)}(a) x_{1}+G_{k}^{(n)}(-a) x_{2} \geq 0,
\end{array}
$$

where $k=0,1, \cdots, p$ and $x_{j} \geq 0, j=1, \cdots, 6$.

To compute bounds on $M(n)$, we found solutions of the SDP problem (2.3)(2.6), restricting our calculation to the case $p=5$. In Table 3 we list the values of SDP bounds for all possible angles except the angle $\arccos \frac{1}{3}$ which is not included because of (1.2) (note that the SDP bounds for other angles are much greater than $2(n-1))$. The column labelled 'max' refers to the maximum of the SDP bounds among all possible angles. The last column in the table gives the value of the angle for which the maximum is attained.

Some comments on the tables are in order. Observe that $M_{1 / 5}=276$ for $23 \leq n \leq 60$. Combined with the results of [10], this implies that $M(n)=276$ for $23 \leq n \leq 41$ and $M(43)=344$. The case $n=42$ remains open since we only obtain that $276 \leq M(42) \leq 288$ for the angle $\arccos 1 / 7$.

Improvements of the Gerzon upper bound (1.1) are obtained for $n \leq 136$. The last 3 entries in Table 3 produced no improvements, and are marked by an asterisk because of that. Similarly, the SDP problem yielded no improvements for higher dimensions.

An interesting, unexplained observation regarding this table is that the SDP bound for $M_{\alpha}(n)$ has long stable ranges for dimensions starting with the value $n=d^{2}-2$, where $d$ is an odd integer and $\alpha=1 / d$. For instance, one such region begins with $d=5$, another with $d=7$. The same phenomenon can observed for $d=9$ where the SDP value $M_{\alpha}(n) \leq 3160$ is obtained for all values of $n$ satisfying $79 \leq n \leq 227$ and for $d=11$ where the value 7140 appears for all $n, 119 \leq n \leq 347$.

Note that the SDP bound gives the same value as the Gerzon bound for $n=$ 47,79 and 119, and that these three dimensions are of the form $n=(2 k-1)^{2}-2$, where $k \geq 2$ is a positive integer. Bannai, Munemasa, and Venkov [2] showed that for $n=47,79$ the maximum possible size $M(n)$ cannot attain this value while the case $n=119$ is still open. The result of $[2$ relies on the fact that an equiangular line set in $\mathbb{R}^{n}$ with cardinality $\frac{n(n+1)}{2}$ gives rise to a spherical two-distance set of size $(n-1)(n+2) / 2$ in $\mathbb{R}^{n-1}$, and such sets are related to tight spherical 4-designs whose existence can be sometimes ruled out. 


\begin{tabular}{|c|c|c|c|c|c|c|c|c|c|}
\hline$n$ & $1 / 5$ & $1 / 7$ & $1 / 9$ & $1 / 11$ & $1 / 13$ & $1 / 15$ & $\max$ & Gerzon & angle \\
\hline 22 & 176 & 39 & 29 & 26 & 25 & 24 & 176 & 253 & $1 / 5$ \\
\hline 23 & 276 & 42 & 31 & 28 & 26 & 25 & 276 & 276 & $1 / 5$ \\
\hline 24 & 276 & 46 & 33 & 29 & 27 & 26 & 276 & 300 & $1 / 5$ \\
\hline 25 & 276 & 50 & 35 & 31 & 29 & 28 & 276 & 325 & $1 / 5$ \\
\hline 26 & 276 & 54 & 37 & 32 & 30 & 29 & 276 & 351 & $1 / 5$ \\
\hline 27 & 276 & 58 & 40 & 34 & 31 & 30 & 276 & 378 & $1 / 5$ \\
\hline 28 & 276 & 64 & 42 & 36 & 33 & 31 & 276 & 406 & $1 / 5$ \\
\hline 29 & 276 & 69 & 44 & 37 & 34 & 33 & 276 & 435 & $1 / 5$ \\
\hline 30 & 276 & 75 & 47 & 39 & 36 & 34 & 276 & 465 & $1 / 5$ \\
\hline 31 & 276 & 82 & 49 & 41 & 37 & 35 & 276 & 496 & $1 / 5$ \\
\hline 32 & 276 & 90 & 52 & 43 & 39 & 37 & 276 & 528 & $1 / 5$ \\
\hline 33 & 276 & 99 & 55 & 45 & 40 & 38 & 276 & 561 & $1 / 5$ \\
\hline 34 & 276 & 108 & 57 & 46 & 42 & 39 & 276 & 595 & $1 / 5$ \\
\hline 35 & 276 & 120 & 60 & 48 & 43 & 41 & 276 & 630 & $1 / 5$ \\
\hline 36 & 276 & 132 & 64 & 50 & 45 & 42 & 276 & 666 & $1 / 5$ \\
\hline 37 & 276 & 148 & 67 & 52 & 47 & 44 & 276 & 703 & $1 / 5$ \\
\hline 38 & 276 & 165 & 70 & 54 & 48 & 45 & 276 & 741 & $1 / 5$ \\
\hline 39 & 276 & 187 & 74 & 57 & 50 & 46 & 276 & 780 & $1 / 5$ \\
\hline 40 & 276 & 213 & 78 & 59 & 52 & 48 & 276 & 820 & $1 / 5$ \\
\hline 41 & 276 & 246 & 82 & 61 & 53 & 49 & 276 & 861 & $1 / 5$ \\
\hline 42 & 276 & 288 & 86 & 63 & 55 & 51 & 288 & 903 & $1 / 7$ \\
\hline 43 & 276 & 344 & 90 & 66 & 57 & 52 & 344 & 946 & $1 / 7$ \\
\hline 44 & 276 & 422 & 95 & 68 & 59 & 54 & 422 & 990 & $1 / 7$ \\
\hline 45 & 276 & 540 & 100 & 71 & 60 & 56 & 540 & 1035 & $1 / 7$ \\
\hline 46 & 276 & 736 & 105 & 73 & 62 & 57 & 736 & 1081 & $1 / 7$ \\
\hline 47 & 276 & 1128 & 110 & 76 & 64 & 59 & 1128 & 1128 & $1 / 7$ \\
\hline 48 & 276 & 1128 & 116 & 78 & 66 & 60 & 1128 & 1176 & $1 / 7$ \\
\hline 49 & 276 & 1128 & 122 & 81 & 68 & 62 & 1128 & 1225 & $1 / 7$ \\
\hline 50 & 276 & 1128 & 129 & 84 & 70 & 64 & 1128 & 1275 & $1 / 7$ \\
\hline 51 & 276 & 1128 & 136 & 87 & 72 & 65 & 1128 & 1326 & $1 / 7$ \\
\hline 52 & 276 & 1128 & 143 & 90 & 74 & 67 & 1128 & 1378 & $1 / 7$ \\
\hline 53 & 276 & 1128 & 151 & 93 & 76 & 69 & 1128 & 1431 & $1 / 7$ \\
\hline 54 & 276 & 1128 & 160 & 96 & 78 & 70 & 1128 & 1485 & $1 / 7$ \\
\hline 55 & 276 & 1128 & 169 & 100 & 81 & 72 & 1128 & 1540 & $1 / 7$ \\
\hline 56 & 276 & 1128 & 179 & 103 & 83 & 74 & 1128 & 1596 & $1 / 7$ \\
\hline 57 & 276 & 1128 & 190 & 106 & 85 & 76 & 1128 & 1653 & $1 / 7$ \\
\hline 58 & 276 & 1128 & 201 & 110 & 87 & 77 & 1128 & 1711 & $1 / 7$ \\
\hline 59 & 276 & 1128 & 214 & 114 & 90 & 79 & 1128 & 1770 & $1 / 7$ \\
\hline 60 & 276 & 1128 & 228 & 118 & 92 & 81 & 1128 & 1830 & $1 / 7$ \\
\hline 61 & 279 & 1128 & 244 & 122 & 94 & 83 & 1128 & 1891 & $1 / 7$ \\
\hline 62 & 290 & 1128 & 261 & 126 & 97 & 85 & 1128 & 1953 & $1 / 7$ \\
\hline 63 & 301 & 1128 & 280 & 130 & 99 & 87 & 1128 & 2016 & $1 / 7$ \\
\hline 64 & 313 & 1128 & 301 & 134 & 102 & 89 & 1128 & 2080 & $1 / 7$ \\
\hline 65 & 326 & 1128 & 325 & 139 & 105 & 91 & 1128 & 2145 & $1 / 7$ \\
\hline 66 & 339 & 1128 & 352 & 144 & 107 & 92 & 1128 & 2211 & $1 / 7$ \\
\hline 67 & 353 & 1128 & 382 & 148 & 110 & 94 & 1128 & 2278 & $1 / 7$ \\
\hline 68 & 367 & 1128 & 418 & 153 & 113 & 97 & 1128 & 2346 & $1 / 7$ \\
\hline 69 & 382 & 1128 & 460 & 159 & 115 & 99 & 1128 & 2415 & $1 / 7$ \\
\hline 70 & 398 & 1128 & 509 & 164 & 118 & 101 & 1128 & 2485 & $1 / 7$ \\
\hline 71 & 416 & 1128 & 568 & 170 & 121 & 103 & 1128 & 2556 & $1 / 7$ \\
\hline 72 & 434 & 1128 & 640 & 176 & 124 & 105 & 1128 & 2628 & $1 / 7$ \\
\hline 73 & 453 & 1128 & 730 & 182 & 127 & 107 & 1128 & 2701 & $1 / 7$ \\
\hline 74 & 473 & 1128 & 845 & 188 & 130 & 109 & 1128 & 2775 & $1 / 7$ \\
\hline 75 & 494 & 1128 & 1000 & 195 & 134 & 112 & 1128 & 2850 & $1 / 7$ \\
\hline 76 & 517 & 1128 & 1216 & 202 & 137 & 114 & 1216 & 2926 & $1 / 9$ \\
\hline 77 & 542 & 1128 & 1540 & 210 & 140 & 116 & 1540 & 3003 & $1 / 9$ \\
\hline 78 & 568 & 1128 & 2080 & 217 & 144 & 118 & 2080 & 3081 & $1 / 9$ \\
\hline 79 & 596 & 1128 & 3160 & 225 & 147 & 121 & 3160 & 3160 & $1 / 9$ \\
\hline 80 & 626 & 1128 & 3160 & 234 & 151 & 123 & 3160 & 3240 & $1 / 9$ \\
\hline 81 & 658 & 1128 & 3160 & 243 & 154 & 126 & 3160 & 3321 & $1 / 9$ \\
\hline 82 & 693 & 1128 & 3160 & 252 & 158 & 128 & 3160 & 3403 & $1 / 9$ \\
\hline
\end{tabular}




\begin{tabular}{|c|c|c|c|c|c|c|c|c|c|}
\hline$n$ & $1 / 5$ & $1 / 7$ & $1 / 9$ & $1 / 11$ & $1 / 13$ & $1 / 15$ & $\max$ & Gerzon & angle \\
\hline 82 & 693 & 1128 & 3160 & 252 & 158 & 128 & 3160 & 3403 & $1 / 9$ \\
\hline 83 & 731 & 1128 & 3160 & 262 & 162 & 130 & 3160 & 3486 & $1 / 9$ \\
\hline 84 & 772 & 1128 & 3160 & 272 & 166 & 133 & 3160 & 3570 & $1 / 9$ \\
\hline 85 & 816 & 1128 & 3160 & 283 & 170 & 136 & 3160 & 3655 & $1 / 9$ \\
\hline 86 & 866 & 1128 & 3160 & 294 & 174 & 138 & 3160 & 3741 & $1 / 9$ \\
\hline 87 & 920 & 1128 & 3160 & 307 & 178 & 141 & 3160 & 3828 & $1 / 9$ \\
\hline 88 & 979 & 1128 & 3160 & 320 & 182 & 143 & 3160 & 3916 & $1 / 9$ \\
\hline 89 & 1046 & 1128 & 3160 & 333 & 186 & 146 & 3160 & 4005 & $1 / 9$ \\
\hline 90 & 1120 & 1128 & 3160 & 348 & 191 & 149 & 3160 & 4095 & $1 / 9$ \\
\hline 91 & 1203 & 1128 & 3160 & 364 & 196 & 152 & 3160 & 4186 & $1 / 9$ \\
\hline 92 & 1298 & 1128 & 3160 & 380 & 200 & 154 & 3160 & 4278 & $1 / 9$ \\
\hline 93 & 1406 & 1128 & 3160 & 398 & 205 & 157 & 3160 & 4371 & $1 / 9$ \\
\hline 94 & 1515 & 1128 & 3160 & 417 & 210 & 160 & 3160 & 4465 & $1 / 9$ \\
\hline 95 & 1556 & 1128 & 3160 & 438 & 215 & 163 & 3160 & 4560 & $1 / 9$ \\
\hline 96 & 1599 & 1128 & 3160 & 460 & 220 & 166 & 3160 & 4656 & $1 / 9$ \\
\hline 97 & 1644 & 1128 & 3160 & 485 & 226 & 169 & 3160 & 4753 & $1 / 9$ \\
\hline 98 & 1691 & 1128 & 3160 & 511 & 231 & 172 & 3160 & 4851 & $1 / 9$ \\
\hline 99 & 1739 & 1128 & 3160 & 540 & 237 & 176 & 3160 & 4950 & $1 / 9$ \\
\hline 100 & 1790 & 1128 & 3160 & 571 & 243 & 179 & 3160 & 5050 & $1 / 9$ \\
\hline 101 & 1842 & 1128 & 3160 & 606 & 249 & 182 & 3160 & 5151 & $1 / 9$ \\
\hline 102 & 1897 & 1128 & 3160 & 644 & 255 & 185 & 3160 & 5253 & $1 / 9$ \\
\hline 103 & 1954 & 1128 & 3160 & 686 & 262 & 189 & 3160 & 5356 & $1 / 9$ \\
\hline 104 & 2014 & 1128 & 3160 & 734 & 268 & 192 & 3160 & 5460 & $1 / 9$ \\
\hline 105 & 2077 & 1128 & 3160 & 787 & 275 & 196 & 3160 & 5565 & $1 / 9$ \\
\hline 106 & 2142 & 1128 & 3160 & 848 & 282 & 199 & 3160 & 5671 & $1 / 9$ \\
\hline 107 & 2211 & 1128 & 3160 & 917 & 289 & 203 & 3160 & 5778 & $1 / 9$ \\
\hline 108 & 2282 & 1128 & 3160 & 997 & 297 & 206 & 3160 & 5886 & $1 / 9$ \\
\hline 109 & 2358 & 1128 & 3160 & 1090 & 305 & 210 & 3160 & 5995 & $1 / 9$ \\
\hline 110 & 2437 & 1128 & 3160 & 1200 & 313 & 214 & 3160 & 6105 & $1 / 9$ \\
\hline 111 & 2521 & 1128 & 3160 & 1332 & 321 & 218 & 3160 & 6216 & $1 / 9$ \\
\hline 112 & 2609 & 1128 & 3160 & 1493 & 330 & 222 & 3160 & 6328 & $1 / 9$ \\
\hline 113 & 2702 & 1128 & 3160 & 1695 & 339 & 226 & 3160 & 6441 & $1 / 9$ \\
\hline 114 & 2800 & 1128 & 3160 & 1954 & 348 & 230 & 3160 & 6555 & $1 / 9$ \\
\hline 115 & 2904 & 1128 & 3160 & 2300 & 357 & 234 & 3160 & 6670 & $1 / 9$ \\
\hline 116 & 3015 & 1128 & 3160 & 2784 & 367 & 238 & 3160 & 6786 & $1 / 9$ \\
\hline 117 & 3132 & 1128 & 3160 & 3510 & 378 & 242 & 3510 & 6903 & $1 / 11$ \\
\hline 118 & 3257 & 1128 & 3160 & 4720 & 388 & 247 & 4720 & 7021 & $1 / 11$ \\
\hline 119 & 3390 & 1128 & 3160 & 7140 & 399 & 251 & 7140 & 7140 & $1 / 11$ \\
\hline 120 & 3532 & 1128 & 3160 & 7140 & 411 & 256 & 7140 & 7260 & $1 / 11$ \\
\hline 121 & 3684 & 1128 & 3160 & 7140 & 423 & 260 & 7140 & 7381 & $1 / 11$ \\
\hline 122 & 3848 & 1128 & 3160 & 7140 & 436 & 265 & 7140 & 7503 & $1 / 11$ \\
\hline 123 & 4024 & 1128 & 3160 & 7140 & 449 & 270 & 7140 & 7626 & $1 / 11$ \\
\hline 124 & 4214 & 1128 & 3160 & 7140 & 462 & 275 & 7140 & 7750 & $1 / 11$ \\
\hline 125 & 4419 & 1128 & 3160 & 7140 & 477 & 280 & 7140 & 7875 & $1 / 11$ \\
\hline 126 & 4643 & 1128 & 3160 & 7140 & 492 & 285 & 7140 & 8001 & $1 / 11$ \\
\hline 127 & 4887 & 1128 & 3160 & 7140 & 508 & 290 & 7140 & 8128 & $1 / 11$ \\
\hline 128 & 5153 & 1128 & 3160 & 7140 & 524 & 295 & 7140 & 8256 & $1 / 11$ \\
\hline 129 & 5447 & 1128 & 3160 & 7140 & 541 & 301 & 7140 & 8385 & $1 / 11$ \\
\hline 130 & 5770 & 1128 & 3160 & 7140 & 560 & 306 & 7140 & 8515 & $1 / 11$ \\
\hline 131 & 6130 & 1128 & 3160 & 7140 & 579 & 312 & 7140 & 8646 & $1 / 11$ \\
\hline 132 & 6531 & 1130 & 3160 & 7140 & 599 & 317 & 7140 & 8778 & $1 / 11$ \\
\hline 133 & 6982 & 1158 & 3160 & 7140 & 620 & 323 & 7140 & 8911 & $1 / 11$ \\
\hline 134 & 7493 & 1187 & 3160 & 7140 & 643 & 329 & 7493 & 9045 & $1 / 5$ \\
\hline 135 & 8075 & 1218 & 3160 & 7140 & 667 & 336 & 8075 & 9180 & $1 / 5$ \\
\hline 136 & 8747 & 1249 & 3160 & 7140 & 692 & 342 & 8747 & 9316 & $1 / 5$ \\
\hline *137 & 9528 & 1282 & 3160 & 7140 & 719 & 348 & 9528 & 9453 & $1 / 5$ \\
\hline *138 & 10450 & 1315 & 3160 & 7140 & 747 & 355 & 10450 & 9591 & $1 / 5$ \\
\hline *139 & 11553 & 1350 & 3160 & 7140 & 778 & 362 & 11553 & 9730 & $1 / 5$ \\
\hline
\end{tabular}

TABLE 3. Values of the SDP bound on $M(n), 22 \leq n \leq 139$ 
Based on the earlier results and our calculations, we make the following

CONJECTURE: There exist 1128 equiangular lines in $\mathbb{R}^{48}$ with angle arccos $(1 / 7)$ and 3160 equiangular lines in $\mathbb{R}^{80}$ with angle arccos $(1 / 9)$.

If this conjecture is true, then $M(n)=1128$ for $48 \leq n \leq 75$ and $M(n)=3160$ for $80 \leq n \leq 116$.

\section{Tight spherical designs of harmonic index 4 and equiangular lines}

Definition 3.1. Let $t$ be a natural number. A finite subset $X$ of the unit sphere $S^{n-1}$ is called a spherical $t$-design if, for any polynomial $f(x)=f\left(x_{1}, x_{2}, \ldots, x_{n}\right)$ of degree at most $t$, the following equality holds :

$$
\frac{1}{\left|S^{n-1}\right|} \int_{S^{n-1}} f(x) d \sigma(x)=\frac{1}{|X|} \sum_{x \in X} f(x) .
$$

A spherical $t$-design is called tight if it attains the LP bound of [7, also called the absolute bound.

An equivalent definition of spherical designs can be given in terms of harmonic polynomials. Let $\operatorname{Harm}_{t}\left(\mathbb{R}^{n}\right)$ be the set of homogeneous harmonic polynomials of degree $t$ on $\mathbb{R}^{n}$. Then the set $X$ is a spherical design [7] if

$$
\sum_{x \in X} f(x)=0 \quad \forall f(x) \in \operatorname{Harm}_{j}\left(\mathbb{R}^{n}\right), 1 \leq j \leq t .
$$

The following definition was recently proposed by Bannai, Okuda, and Tagami 3]: A spherical design of harmonic index $t$ is a finite subset $X \subset S^{n-1}$ such that

$$
\sum_{x \in X} f(x)=0 \quad \forall f(x) \in \operatorname{Harm}_{t}\left(\mathbb{R}^{n}\right) .
$$

An LP bound for spherical designs of harmonic index $t$ was derived in [3]. Similarly, if this bound is attained, then the design is called tight. Our interest in tight spherical designs of a fixed harmonic index is motivated by a result in $[\mathbf{3}$. which shows that a tight design of index 4 gives rise to an equiangular line set in $\mathbb{R}^{n}$ with angle $\arccos a=\sqrt{3 /(n+4)}$. Since $a=\frac{1}{2 k-1}$ for some integer $k \geq 2$, we find that $n=3(2 k-1)^{2}-4$. These considerations motivate the following result.

Theorem 3.2. Let $n=3(2 k-1)^{2}-4, k \geq 2$. The cardinality $N$ of any equiangular line set in $\mathbb{R}^{n}$ with inner product $a=1 /(2 k-1)$ satisfies the inequality

$$
|S| \leq \frac{(n+1)(n+2)}{6}
$$

Proof. To prove this result we use the LP bound of $[\mathbf{7}$ that has the following form: Let $T \subset[-1,1]$. Let $S=\left\{x_{1}, x_{2}, \ldots, x_{N}\right\}$ be a set of unit vectors in $\mathbb{R}^{n}$ such $\left\langle x_{i}, x_{j}\right\rangle \in T \cup\{1\}$. Let $f(t)=\sum_{k} f_{k} G_{k}^{n}(t)$ be a polynomial such that $f_{0}>0, f_{k} \geq$ $0, k \geq 1$ and that $f(t) \leq 0$ for all $t \in T$. Then

$$
|S| \leq\left\lfloor\frac{f(1)}{f_{0}}\right\rfloor .
$$

Consider the polynomial

$$
f(t)=\left(t^{2}-a^{2}\right)\left(t^{2}+\frac{a^{2} n+4 a^{2}-6}{n+4}\right) .
$$


Let $X \subset \mathbb{R}^{n}$ be an equiangular line set with inner product $a$. Then $T=\{ \pm a\}$, and $f(t)=0$ for $t \in T$. Computing the Gegenbauer expansion of $f(t)$, we obtain

$$
\begin{aligned}
& f_{0}=-\frac{a^{4} n^{2}+6 a^{2} n\left(a^{2}-1\right)+8 a^{4}-6 a^{2}(n+2)+3}{n^{2}+6 n+8} \\
& f_{1}=f_{2}=f_{3}=0 \\
& f_{4}=\frac{n^{2}-1}{(n+2)(n+4)}
\end{aligned}
$$

We need to check that $f_{0}>0$. Substituting the values of $n$ and $a$, we obtain

$$
f_{0}=\frac{8 k(k-1)}{(2 k-1)^{4}\left(12 k^{2}-12 k+1\right)} \geq 0 \quad \text { for } k \geq 2 .
$$

Thus, $f(t)$ satisfies the conditions of the LP bound, and we obtain

$$
|S| \leq \frac{f(1)}{f_{0}}=\frac{\left(a^{2}-1\right)(n+2)\left(n+a^{2} n+4 a^{2}-2\right)}{a^{4} n^{2}+6 a^{4} n+8 a^{4}-6 a^{2} n-12 a^{2}+3} .
$$

In particular, putting $a=\frac{1}{2 k-1}$ and $n=3(2 k-1)^{2}-4=12 k^{2}-12 k-1$, we obtain

$$
\frac{f(1)}{f_{0}}=\frac{(n+1)(n+2)}{6} \text {. }
$$

This theorem gives infinitely many values of $n$ for which the upper bound $M_{a}(n)$ is strictly less than the Gerzon bound, yielding the asymptotic constant $1 / 6$ for the growth rate of the quantity $M_{\alpha}(n)$ (cf. (1.4)-(1.5)).

REMARK 3.3. Observe that the relative bound (1.3) is an instance of the LP bound (3.2); see 7 . Thus, the SDP bound (2.3) $-(2.6)$ is as strong or stronger than the bound (1.3).

REMARK 3.4. Using SDP, we further show that for some dimensions the LP bound (3.1) cannot be attained. Indeed, for $k=3,4,5$ we obtain the values of the dimension $n=71,143,239$, respectively, and the SDP bound implies that

$$
M_{1 / 5}(71) \leq 416, \quad M_{1 / 7}(143) \leq 1506, \quad M_{1 / 9}(239) \leq 3902,
$$

which is much smaller than the values $876,3480,9640$ obtained from (3.1). Extending these calculations, we have shown that for $k \leq 54$ and $n=3(2 k-1)^{2}-4 \leq 34343$ the SDP bound improves upon the LP bound (3.1).

In conclusion, we note that the value of the maximum in the LP problem for the maximum cardinality of equiangular line sets with a given angle can be explicitly characterized. The LP problem has the following form:

$$
M_{a}(n) \leq \max \left\{1+x_{1}+x_{2}, x_{1} \geq 0, x_{2} \geq 0\right\}
$$

subject to

$$
1+G_{k}^{n}(a) x_{1}+G_{k}^{n}(-a) x_{2} \geq 0 \text { for } k=1,2, \ldots
$$

Theorem 3.5. Let $a \in(0,1)$,

$$
g_{n}=\min _{k \geq 0} \frac{1}{\left|G_{k}^{n}(a)\right|}
$$

where $k$ is even and such that $G_{k}^{n}(a)<0$. Then

$$
M_{a}(n) \leq g_{n}+1,
$$

where the value $g_{n}+1$ is the solution of the LP problem (3.3),(3.4). 
Proof. Let $k$ be even, then $G_{k}^{n}(t)$ is an even function, so inequalities (3.4) take the form

$$
1+G_{k}^{n}(a)\left(x_{1}+x_{2}\right) \geq 0, \quad k=2 m, m \in \mathbb{N} .
$$

These inequalities define a set of half-planes whose boundaries are parallel to the objective function. The inequalities for odd $k$ are bounded by lines that are perpendicular to the boundaries of the even-indexed constraints, and therefore can be disregarded. We conclude that the maximum is attained on the line $1+G_{k}^{n}(a)\left(x_{1}+x_{2}\right)=0$ for some even $k$. The inequalities with $k$ such that $G_{k}^{n}(a) \geq 0$ are trivially satisfied, therefore, we consider only those values of $k$ when $G_{k}^{n}(a)<0$. Eq. (3.6) implies that, for all even $k$,

$$
x_{1}+x_{2} \leq-\frac{1}{G_{k}^{n}(a)}=\frac{1}{\left|G_{k}^{n}(a)\right|} .
$$

This completes the proof.

To give an example of using this theorem, take $n=71$ and $a=\frac{1}{5}$. To find a bound on $M_{a}(n)$, we estimate the quantity $g_{n}$ in (3.5) by computing

$$
\min _{0 \leq k \leq 100} \frac{1}{\left|G_{k}^{(71)}(1 / 5)\right|}
$$

for all even $k$ such that $G_{k}^{(71)}(1 / 5)<0$. The smallest value is obtained for $k=4$, and $G_{4}^{(71)}(1 / 5)=-1 / 875$. Thus, we obtain $M_{1 / 5}(71) \leq 876$. Of course, it could be possible that for greater $k$ we obtain a smaller value of the bound, but this is not supported by our experiments (although we do not have a proof that $k=4$ is the optimal choice).

Experiments also suggest that $k=4$ may be the universal optimal choice for infinitely many values of $n$ and $a$. Indeed, we have

$$
G_{4}^{n}(x)=\frac{(n+2)(n+4) x^{4}-6(n+2) x^{2}+3}{n^{2}-1} .
$$

Taking $n=3(2 t-1)^{2}-4$ and $a=1 /(2 t-1)$, where $t \geq 2$, we obtain the expression

$$
\frac{1}{G_{4}^{n}(a)}+1=2 t(t-1)\left(12 t^{2}-12 t+1\right)=\frac{(n+1)(n+2)}{6}
$$

which coincides with the LP bound (3.1).

Acknowledgement: We thank Peter Casazza, Eiichi Bannai, and Ferenc Szöllösi for insightful discussions on the topics of this paper.

Alexander Barg was supported in part by NSF grants DMS1101697, CCF1217245, and CCF1217894, and by NSA grant H98230-12-1-0260. Wei-Hsuan Yu was supported in part by NSF grant CCF CCF1217245.

\section{References}

[1] C. Bachoc and F. Vallentin, New upper bounds for kissing numbers from semidefinite programming, J. Amer. Math. Soc. 21 (2008), 909-924.

[2] E. Bannai, A. Munemasa, and B. Venkov, The nonexistence of certain tight spherical designs, St. Petersburg Math. J. 16 (2005), 609-625.

[3] E. Bannai, T. Okuda, and M. Tagami, Spherical designs of harmonic index $t$, http://arxiv.org/pdf/1308.5101v1.pdf (August 2013). 
[4] A. Barg and W.-H. Yu, New bounds for spherical two-distance set, Experimental Mathematics, 22, No. 2, (2013), 187-194.

[5] L. Vandenberghe and S. Boyd Semidefinite programming, SIAM Review, 38, No.1 (1996), 49-95.

[6] D. de Caen, Large equiangular sets of lines in Euclidean space, Electron. J. Combin. 7 (2000), Research Paper 55, 3pp.

[7] P. Delsarte, J. M. Goethals, and J. J. Seidel, Spherical codes and designs, Geometriae Dedicata 6 (1977), 363-388.

[8] J. Hanntjes, Equilateral point-sets in elliptic two- and three-dimensional spaces, Nieuw Arch Wisk 22 (1948), 355-362

[9] D. G. Larman, C. A. Rogers, and J. J. Seidel, On two-distance sets in Eeuclidean space, Bull. London Math. Soc. 9 (1977), 261-267.

[10] P. W. H. Lemmens and J. J. Seidel, Equiangular lines, Journal of Algebra 24 (1973), 494-512.

[11] J. H. van Lint and J. J. Seidel Equiangular point sets in elliptic geometry, Proc. Nederl Akad. Wetensch Series A 69 (1966), 335-348.

[12] G. Nebe and B. Venkov, On tight spherical designs, Algebra i Analiz 24 (2012), no. 3, 163171; translation in St. Petersburg Math. J. 24 (2013), no. 3, 485-491

[13] A. Neumaier, Distance matrices, dimension, and conference graphs, Indag. Math., 43 (1981), no. 4, 385-391.

[14] I. J. Schoenberg, Positive definite functions on spheres, Duke Math. J. 9 (1942), 96-107.

[15] M. A. Sustik, J. A. Tropp, I. S. Dhillon and R.W. Heath, Jr., On the existence of equiangular tight frames, Linear Alg. and Applications 426, no. 2-3 (2007), 619-635.

[16] G. Greaves, J. H. Koolen, A. Munemaza, and F. Szöllősi, Equiangular lines in Euclidean spaces, preprint arXiv:1403:2155 (http://arxiv.org/abs/1403.2155).

Dept. of ECE and Inst. for Systems Research, University of Maryland, College

Park, MD, 20742, and Inst. for Problems of Inform. Trans., RAS, Moscow, Russia

E-mail address: abarg@umd.edu

Department of Math. and Inst. for Systems Research, University of Maryland, College Park, MD 20742

E-mail address: mathyu@math.umd.edu 